\title{
Research on Modified Algorithms of Cylindrical External Thread Profile Based on Machine Vision
}

\author{
Chen $\mathrm{JH}^{1,2}$,Zhang $\mathrm{JJ}^{1}$, Gao $\mathrm{RJ}^{1}$, Jiang $\mathrm{CH}^{4}$, Ma R ${ }^{1}$, Qi ZM${ }^{3}$, Jin $\mathrm{H}^{2}$, Zhang $\mathrm{HD}^{2}$, Wang XC ${ }^{2}$ \\ ${ }^{1}$ Institute of Mechanical Engineering of Zhejiang University, Zheda Road, No.38, 310012, Hangzhou, China \\ ${ }^{2}$ Ningbo Institute of Technology of Zhejiang University, Qianhunan Road, No.1, 315100, Ningbo, China \\ ${ }^{3}$ Institute of Engineering of Zhejiang University, Huzhou Street, No.50, 310015, Hangzhou, China \\ ${ }^{4}$ Institute of Mechanical Engineering and Mechanics of Ningbo University, Fenghua Road, No.818, 315211, Ningbo, China
}

\begin{abstract}
In the non-contact detection of thread profile boundary correction, it remains challenging to ensure that the thread axis intersects the CCD camera axis perpendicularly. Here, we addressed this issue using modified algorithms. We established the Cartesian coordinate system according to the spatial geometric relationship of the thread. We used the center of the bottom of the thread as the origin, and the image of the extreme position image was replaced by the image of the approximate extreme position. In addition, we analyzed the relationship between the boundary of the theoretical thread image and the theoretical profile. We calculated the coordinate transformation of the point on the theoretical tooth profile and the coordinate function of the point on the boundary of the theoretical image. At the same time, the extreme value of the function was obtained, and the boundary equation of the theoretical thread image was deduced. The difference equation between the two functions was used to correct the boundary point of the actual thread image, and the fitting results were used to detect the key parameters of the external thread of the cylinder. Further experiment proves that the above algorithm effectively improves the detection accuracy of thread quality, and the detection error of main geometric parameters is reduced by more than $50 \%$.
\end{abstract}

Keywords: Theoretical tooth profile, theoretical image boundary, actual tooth profile, actual image boundary, machine vision, difference equation, screw thread image processing.

\section{INTRODUCTION}

Thread fasteners are common modern industrial products. They are important joint fastening components in the mechanical industry and are used in almost all mechanical equipment. Thread fasteners have become one of the standardized parts due to their wide applications. Many industrial products are composed of a large number of thread fasteners. The geometric parameters of threads, especially the standardization degree of thread profile, have a great impact on the overall quality of products. Therefore, how to measure the geometric parameters of threads in the production process efficiently and accurately is of great significance. Traditional thread gage measurement methods, e.g. three-needle (doubleneedle) measurement method and thread micrometer measurement method, cannot meet the needs of industry because of their slow speed, low accuracy, and single measurement parameters. Therefore, it is very important to develop detection equipment and detection methods that are applicable to different occasions and meet diverse needs.

Machine vision is a branch of artificial intelligence which has developed rapidly in recent years. It is important to apply machine vision in factory workshop to collect images by vision and then analyze and process the collected images. In the production process of many kinds of industry, a large number of repeated processes can be replaced by the detection method which is based on machine vision, through this method, the efficiency and automation of production are greatly improved.

In research papers published by Shen Menghong, Zhang Zhaofeng, and Jiang Zhiping on detection methods [1]-[3], when using machine vision to conduct non-contact detection of thread quality, it is assumed that the axis of the CCD camera and the cross-section of threads are perpendicular to each other in their thread boundary correction algorithms. However, in practice when the tested bolt is clamped on the thread fasteners, it is difficult to ensure that the thread axis completely aligns with the axis of the fixture on the detection platform, which causes the axis of the CCD camera not being perpendicular to the cross-section of threads through the axis. When the image taken by the CCD camera is processed, if the thread boundary is calculated according to the vertical section of the axis of the CCD camera and the thread across the axis, it would inevitably lead to some errors in the calculation results, as in [4], and the quality of the thread detected would not meet the industry requirements. Therefore, it is necessary 
to research on the boundary correction algorithm for cylindrical external threads when the axis is inclined as shown in Fig.2.

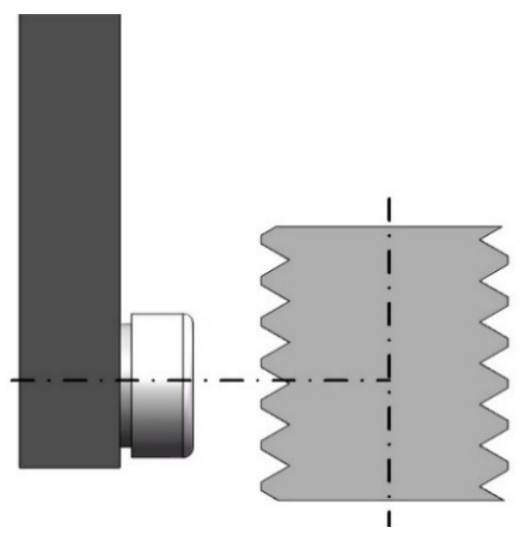

Fig.1. Schematic diagram of CCD camera axis that is perpendicular to the thread axis.

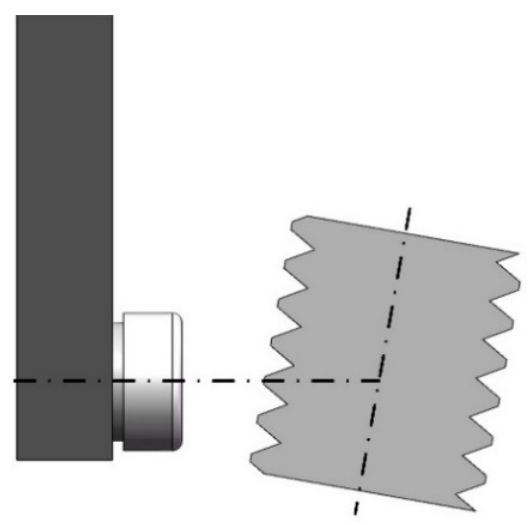

Fig.2. Schematic diagram of CCD camera axis that is not perpendicular to the thread axis.

\section{NON-CONTACT DETECTION PROCESS FOR THREAD BOUNDARY}

In the conventional detection method, as in [5], when the external thread is used for image acquisition, the cylindrical external thread is generally fixed at a certain position by means of a clamp. The outline image of external threads is acquired by a CCD camera, and the screw image is processed accordingly for outline extraction, feature point matching, and others. The geometric relationship in the image is then calculated to obtain the required parameter information.

In this paper, some improvements are made to the external thread detection system. The workpiece clamping table is adjusted to a uniform rotating platform controlled by a brushless motor, as in [6]. The single thread image is modified to a continuous video sequence, and the number of frames needed is intercepted from the video sequence. The following is the detection flowchart using this algorithm.

The acquisition of a video sequence is accomplished by the hardware part of the system. With the threads being rotated at a uniform speed driven by the rotating platform, as in [7] and [8], CCD obtains the complete video sequence of a period of rotation as in [9], and then transmits the collected information to the computer through the image acquisition card. In addition to the traditional CCD camera, light source, image acquisition card, and computer, we need to design a rotating detection platform in the hardware of the system, which will make the measured parts rotate at a uniform speed under the control of a PLC. In this paper, the speed of the worktable is 1 rotation/s and the frame number of the camera is 30 frames/s.

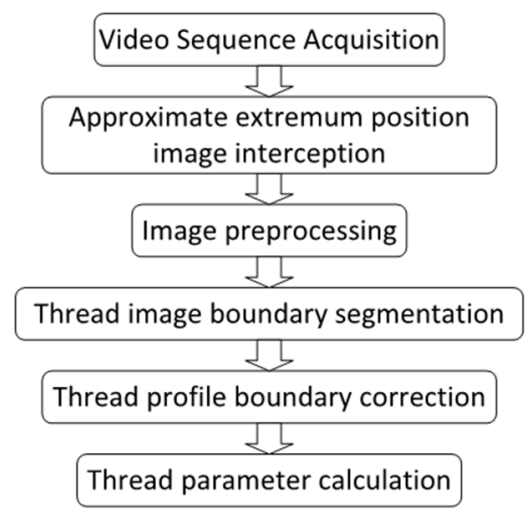

Fig.3. Detection flowchart.

After the acquisition of the video sequence, we first need to intercept the extreme position image, make gray-scale conversion, denoising, enhancement and other processing of the image, and then extract the boundary, as in [10]. We then extract the key points on the image boundary and correct these key points. Finally, we obtain the modified tooth profile for the calculation of thread parameters. When a piece is clamped on a rotating platform, its axis may be at a certain angle to the axis of the platform. The bigger the angle, the greater the impact on the final test results. If the method of manual adjustment is used in clamping, a lot of time will be wasted and the detection efficiency will be reduced, as in [11]. In addition, the manual adjustment method cannot be used in a large-scale setting. Although a fixture with automatic alignment function can be designed, it is still difficult to completely eliminate the angle between the axis of the measured piece and the platform axis. Moreover, the versatility of such a fixture may be limited. Thus, in theory a method that can be used to correct the points on the contour curve in the software will greatly reduce the impact of these problems.

\section{MATHEMATICAL MODEL FOR THE CONSTRUCTION OF CYLINDRICAL EXTERNAL THREAD}

When the thread axis is in an arbitrary position, the screw image of the initial position can be obtained by a CCD camera. At this time, the theoretical coordinate system is established with the center of the thread bottom as the origin. As shown in Fig.4., the angle between OA and $z$ axis is $\lambda$, and the angle between OP and $y$ axis of OA projection on $O x y$ plane is $\mu$. When the worktable rotates at a uniform speed, that is, when the thread axis OA rotates around the $z$ axis, there will be inevitably eight positions, as shown in Fig.5. Thread images near position 3 or 7 can be obtained by 
intercepting the corresponding frames in the thread video sequence. Position 3 and position 7 are called extreme positions. Thread images intercepted are not necessarily images of the OA axis on the $O y z$ plane. The rotational speed is rotation $/ \mathrm{s}$ and the frame number of the camera is 30 frames/s. The position as shown in Fig.5. is the screw image with the smallest angle between the projection of the axis on the $O x y$ plane and the $O y z$ plane. This position is called the approximate extremum position. The angle between the projection of the axis and the $O y z$ plane must be less than 6 degrees. The calculation error is much less than that of the detection algorithm without considering the angle between the thread axis and the $z$ axis. Therefore, the approximate extremum position image can be used. Thread parameters are calculated instead of the use of extreme position image, as in [12]. The occlusion diagram is shown in Fig.6.

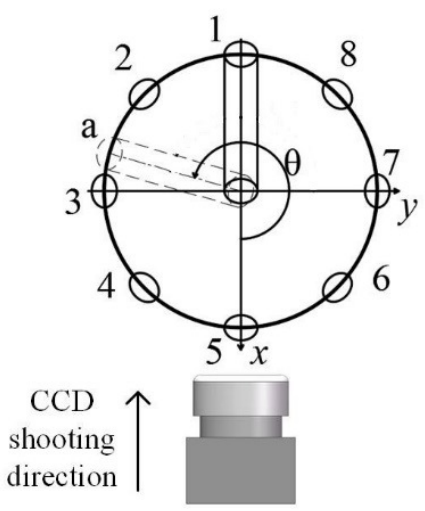

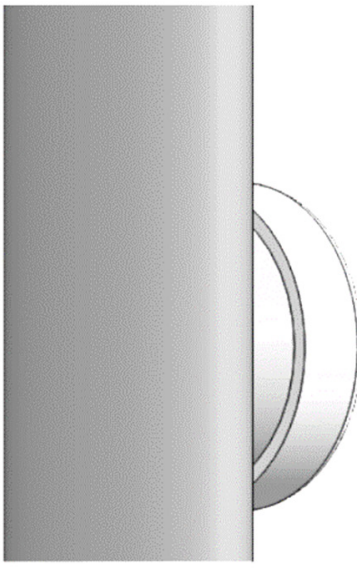

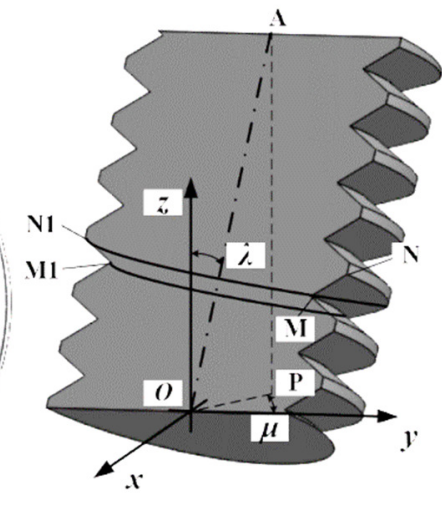

Fig.4. Schematic diagram of the theoretical coordinate system.

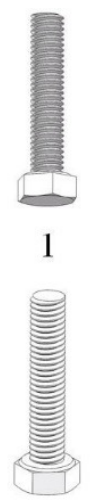

5
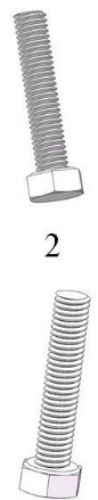

6

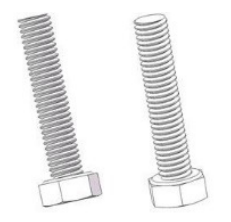

34

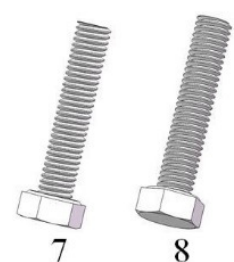

Fig.5. Thread image of CCD camera captured at different positions.

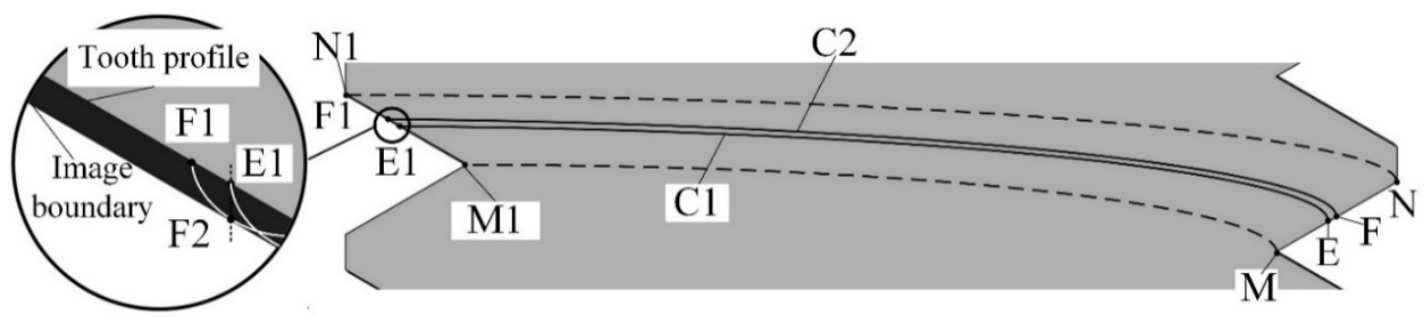

Fig.6. Schematic diagram of thread image occlusion.

However, in the extreme position image, there are some errors between the image boundary and the actual thread tooth profile. The accuracy of thread detection will be directly affected if the thread parameters are measured directly using the captured image boundary. As shown in Fig.6., the point E1 on the tooth profile can be obtained by rotating around the axis OA by 180 degrees along the helix $\mathrm{C} 1$, while the point F1 on the tooth profile $\mathrm{MN}$ can be obtained by rotating another point $\mathrm{F}$ around the axis by 180 degrees along the helix $\mathrm{C} 2$.But this cylindrical spiral will intersect at point $\mathrm{F} 2$ on a straight line that passes E1 and is parallel to the OA when the angle of rotation is less than 180 degrees. That is, F2 is the occlusion point of point $\mathrm{E} 1$ when it rotates along the spiral elevation angle from the point $\mathrm{F}$ on the tooth profile $\mathrm{MN}$. Either point on the tooth profile of E1 will be occluded by the point on the $\mathrm{MN}$ of the tooth profile when it rotates, so we get the curve where F2 is located, which is the image boundary. The line of F1 is the tooth profile needed in practical calculation. In order to improve the accuracy of thread detection, it is necessary to calculate the difference equation between the tooth profile and the image boundary. The difference equation is used to correct the points on the boundary of the captured image. The corrected points are then fitted with MATLAB to obtain the profile of the tooth for calculating the relevant parameters of the cylindrical external threads. 
Since the external threads of the cylinder can be regarded as parallel to each other in the axial direction, the difference equation between the theoretical tooth profile and the theoretical image boundary is equal, and the thread in the first pitch is calculated with $\mathrm{MN}$ as the axis. When the coordinate axis coincides with the coordinate line of the thread, point $\mathrm{E}$ and point $\mathrm{F}$ are two points on the contour of the tooth. Setting the coordinates of point $\mathrm{E}\left(x_{E}, y_{E}, z_{E}\right)$ when $\mathrm{OA}$ is vertically coincident with the $\mathrm{z}$ axis, the coordinates of point E are:

$$
\left\{\begin{array}{l}
x_{E}=\rho \times \cos \theta \\
y_{E}=\rho \times \sin \theta \\
z_{E}=\frac{p \theta}{2 \pi}
\end{array}\right.
$$

$\rho$ is the distance between point $\mathrm{E}$ and axis, and $\theta$ is the angle of projection of the line between point $\mathrm{E}$ and point $\mathrm{O}$ on the Oxy plane when rotating around the $\mathrm{z}$ axis, and $\mathrm{p}$ is the pitch of the cylindrical thread.

When the axis coincides with the $\mathrm{Z}$ axis and point $\mathrm{E}$ is in the Oyz plane, the coordinates of point $E$ are:

$$
\left\{\begin{array}{l}
x_{E}=0 \\
y_{E}=\rho \\
z_{E}=\frac{p}{4}
\end{array}\right.
$$

When the angle between OA and $z$ axis is $\lambda, \lambda \in(0, \pi / 2)$, which is equivalent to point $\mathrm{E}$ rotating clockwise around the $\mathrm{X}$ axis in $O y z$ plane, and counter-clockwise $(-\lambda)$, at which point E coordinates are:

$$
\left\{\begin{array}{l}
x_{E}=0 \\
y_{E}=\rho \times \cos (-\lambda)-\frac{p}{4} \times \sin (-\lambda) \\
z_{E}=\rho \times \sin (-\lambda)+\frac{p}{4} \times \cos (-\lambda)
\end{array}\right.
$$

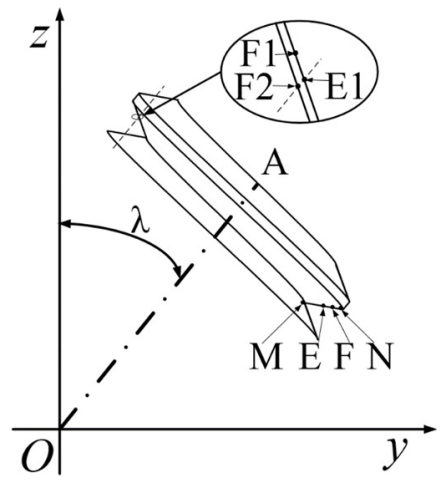

Fig.7. Diagram of screw image boundary occlusion.

As shown in Fig.7., the point E coordinates are:

$$
\left\{\begin{array}{l}
x_{E}=0 \\
y_{E}=\rho \times \cos \lambda+\frac{p}{4} \times \sin \lambda \\
z_{E}=-\rho \times \sin \lambda+\frac{p}{4} \times \cos \lambda
\end{array}\right.
$$

Point $\mathrm{E}$ rotates around axis $\mathrm{OA}$ for 180 degrees to get point E1. The coordinates of point E1 can be expressed as follows:

$$
\left\{\begin{array}{l}
x_{E 1}=0 \\
y_{E 1}=-\rho \times \cos \lambda+\frac{3 p}{4} \times \sin \lambda \\
z_{E 1}=\rho \times \sin \lambda+\frac{3 p}{4} \times \cos \lambda
\end{array}\right.
$$

Point F2 is the point on the edge of the thread image equal to the distance from E1 to the thread axis OA, so the coordinates of point F2 can be expressed as:

$$
\left\{\begin{array}{l}
x_{F 2}=0 \\
y_{F 2}=-\rho \times \cos \lambda+\left(\frac{p}{4}+\mathrm{t} \times \sin \beta+\frac{p \gamma}{2 \pi}\right) \times \sin \lambda \\
z_{F 2}=\rho \times \sin \lambda+\left(\frac{p}{4}+\mathrm{t} \times \sin \beta+\frac{p \gamma}{2 \pi}\right) \times \cos \lambda
\end{array}\right.
$$

Where $\gamma$ is expressed as the angle at which point $\mathrm{F}$ rotates around axis $\mathrm{OA}$, $t$ is the distance from point $\mathrm{F} 2$ to point $\mathrm{E} 1$ on the thread profile, and $\beta$ is the half-angle of the thread.

By subtracting $Z_{F 2}$ from $Z_{E 1}$, the difference between $\mathrm{E} 1$ and $\mathrm{F} 2$ in the $\mathrm{z}$ axis direction is obtained

$$
\Delta \mathrm{z}=z_{E 1}-z_{F 2}=\left(\frac{p}{2}-\frac{p \gamma}{2 \pi}-\mathrm{t} \times \sin \beta\right) \times \cos \lambda
$$

The difference equation between $\mathrm{E} 1$ and F2 in the axis direction is obtained by angle transformation. The difference equation in the axis direction is as follows:

$$
\begin{gathered}
\Delta=\frac{\Delta \mathrm{z}}{\sin \left(\frac{\pi}{2}-\lambda\right)} \\
=\left(\frac{p}{2}-\frac{p \gamma}{2 \pi}-\mathrm{t} \times \sin \beta\right) \times \cos \lambda / \sin \left(\frac{\pi}{2}-\lambda\right) \\
=\left(\frac{p}{2}-\frac{p \gamma}{2 \pi}-\mathrm{t} \times \sin \beta\right)
\end{gathered}
$$

It can be simplified to:

$$
\Delta=\frac{p \times(\pi-\gamma)}{2 \pi}-\mathrm{t} \times \sin \beta
$$

As long as the relationship between $\gamma$ and the points on the tooth profile is known, the definite expression of the difference equation can be obtained, and the image boundary can be corrected by the use of difference equation feedback.

The tooth profile angle of the thread is simplified to triangular tooth profile, and the half-angle of the known thread profile is $\beta$. As shown in Fig.7., it can be seen that the distance from point $\mathrm{E}$ to axis $\mathrm{OA}$ is $\rho$, and the distance from point $\mathrm{F}$ to axis is $\rho+\mathrm{t} \times \cos \beta$. After point $\mathrm{F}$ rotates the $\gamma$ angle around the axis, the point F2 equal to the distance from E1 to axis can be obtained:

$$
(\rho+\mathrm{t} \times \cos \beta) \times \cos \gamma=\rho
$$


So,

$$
t=\frac{\frac{\rho}{\cos \gamma}-\rho}{\cos \beta}
$$

The ordinates of $\mathrm{F} 2$ can also be expressed as:

$$
Z_{F 2}=\rho \times \sin \lambda+\left(\frac{p}{4}+\mathrm{t} \times \sin \beta+\frac{p \gamma}{2 \pi}\right) \times \cos \lambda
$$

That is:

$$
Z_{F 2}=\rho \times \sin \lambda+\left(\frac{p}{4}+\frac{\frac{\rho}{\cos \gamma}-\rho}{\cos \beta} \times \sin \beta+\frac{p \gamma}{2 \pi}\right) \times \cos \lambda
$$

In order to obtain the image boundary of threads, the minimum value of $Z_{F 2}$ is required. In the formula above, $Z_{F 2}$ is a function of $\gamma$, and the derivation of $Z_{F 2}$ can be obtained as follows:

$$
Z_{F 2}{ }^{\prime}=\frac{\sin \gamma}{\cos ^{2} \gamma} \times \rho \times \tan \beta \times \cos \lambda+\frac{p}{2 \pi} \times \cos \lambda
$$

Let $z_{F 2}{ }^{\prime}=0$, we can get:

$$
\sin \gamma=\frac{2 \pi \times \rho \times \tan \beta \pm \sqrt{4 \pi^{2} \times \tan ^{2} \beta+4 p^{2}}}{2 p}
$$

$\gamma \in\left(\frac{\pi}{2}, \pi\right]$, therefore,

$$
\begin{gathered}
\sin \gamma=\frac{2 \pi \times \rho \times \tan \beta-\sqrt{4 \pi^{2} \times \rho^{2} \times \tan ^{2} \beta+4 p^{2}}}{2 p} \\
\gamma=\arcsin \frac{2 \pi \times \rho \times \tan \beta-\sqrt{4 \pi^{2} \times \rho^{2} \times \tan ^{2} \beta+4 p^{2}}}{2 p}
\end{gathered}
$$

So the difference equation is that:

$$
\begin{aligned}
& \Delta= \\
& \frac{p}{2 \pi}\left(\pi-\arcsin \frac{2 \pi \times \rho \times \tan \beta-\sqrt{4 \pi^{2} \times \rho^{2} \times \tan ^{2} \beta+4 p^{2}}}{2 p}\right)-\left(\frac{\rho}{\cos \left(\arcsin \frac{2 \pi \times \rho \times \tan \beta-\sqrt{4 \pi^{2} \times \rho^{2} \times \tan ^{2} \beta+4 p^{2}}}{2 p}\right)}-1\right) \times \tan \beta
\end{aligned}
$$

By substituting the boundary points of thread image into the difference equation, the corresponding points on the actual thread profile can be obtained. These points are fitted by MATLAB, as in [13], and the straight line obtained is the thread profile equation.

\section{EXPERIMENTAL VERIFICATION OF BOUNDARY MODIFICATION MODEL FOR CYLINDRICAL EXTERNAL THREADS}

According to the detection method introduced above, a cylindrical external thread detection platform was designed, similar to that in [14]. Cylindrical external thread detection platform is mainly composed of a CCD camera, light source, rotary table, PLC, computer, and output equipment. The rotary table drives the measured cylindrical external thread workpiece to rotate uniformly on the worktable under the control of the PLC. The video sequence acquired by the CCD camera during rotation is imported into the computer for processing and displayed in the output device. The overall composition of the workbench system is shown in Fig.8.

The video sequence is intercepted in MATLAB to obtain the approximate extremum position image suitable for processing. The extremum position image is used instead of the extremum position image. The subpixel image boundary of the workpiece is extracted by the subpixel edge extraction method. The extracted thread image boundary is shown in the figure. The point on the thread image boundary is corrected by the cylindrical thread profile correction algorithm based on machine vision. Correction is made to get the corresponding points on the profile of the threaded tooth. The least square method is used to fit these points. The straight line is the actual profile of the tooth. The parameters of the cylindrical external thread are measured and calculated using the equation.

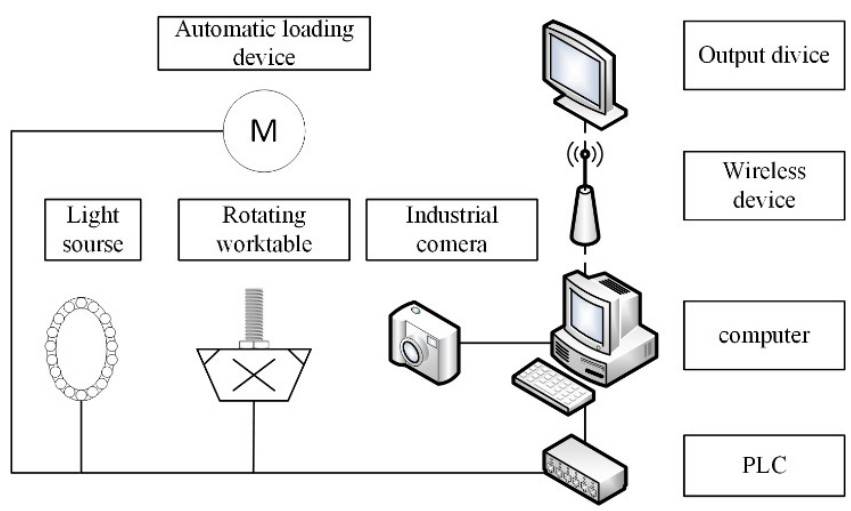

Fig.8. The overall composition of the system.

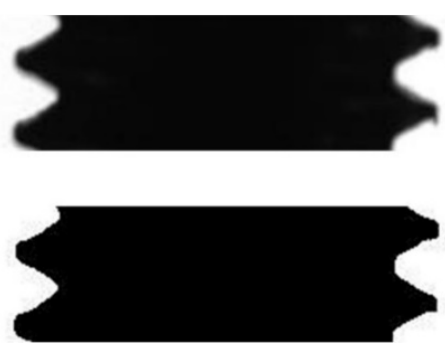

Fig.9. Thread image of extremum position and thread image after pre-processing. 


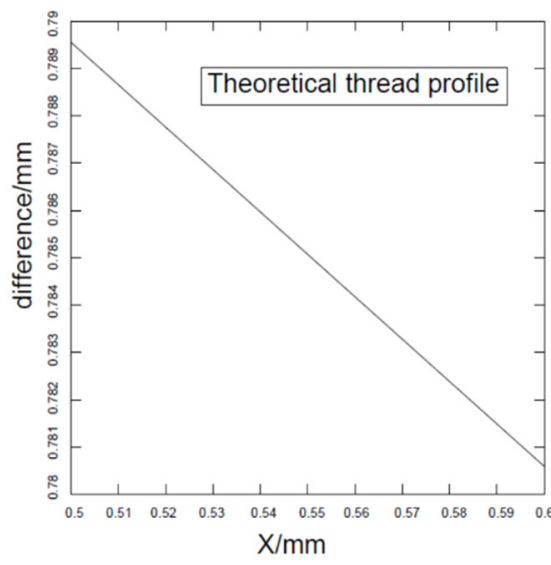

Fig.10. M6 difference equation curve.

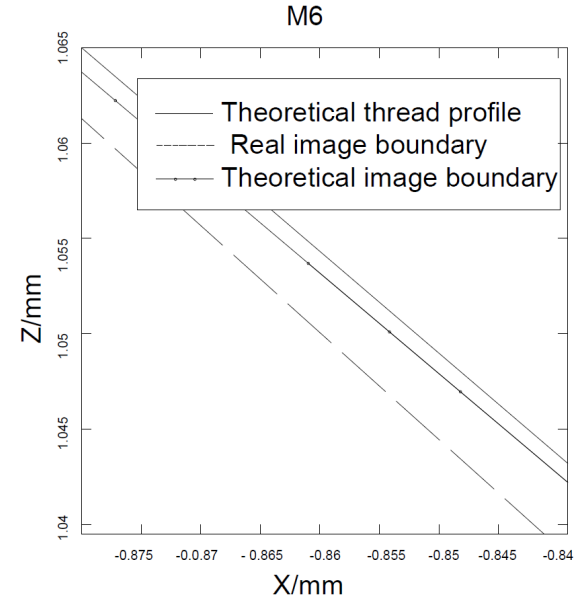

Fig.11. M6 image boundary correction.

Table 1. M6 thread parameter measurement results.

\begin{tabular}{cccccc}
\hline \multirow{2}{*}{ thread parameter } & \multirow{2}{*}{$\begin{array}{c}\text { nominal } \\
\text { size }\end{array}$} & \multicolumn{2}{c}{ projection curve } & \multicolumn{2}{c}{ axis correction curve } \\
\cline { 3 - 5 } & & measured value & deviation & measured value & deviation \\
\hline major diameter & 6.000 & 6.048 & 0.048 & 6.021 & 0.021 \\
pitch diameter & 5.350 & 5.400 & 0.050 & 5.373 & 0.023 \\
minor diameter & 4.917 & 4.941 & 0.024 & 4.887 & 0.003 \\
pitch & 1.00 & 0.984 & 0.016 & 0.987 & 0.013 \\
angle & $60^{\circ}$ & $60.832^{\circ}$ & $0.832^{\circ}$ & $60.012^{\circ}$ & $0.012^{\circ}$ \\
\hline
\end{tabular}

In order to verify the accuracy of the designed algorithm, the thread with nominal diameter of M6 is set at $\lambda$ of 30 degrees. The extremum image was imported into MATLAB for the experiment. The difference equation curve was drawn in MATLAB and the curve was used to modify the boundary of the thread image. Finally, the modified tooth profile was obtained and used to calculate the thread parameters.

The thread contour was modified by the algorithm proposed in this paper, and the thread parameters were calculated by the modified thread image. The final measurement results of the thread with nominal diameter of M6 compared with projection curve as in [1] are shown in Table 1.

The experimental results showed that for the three parameters of major diameter, pitch diameter, minor diameter and thread angle, the projection curve as in [15] was corrected with the axis angle of the measured part, and then the parameters were calculated. Compared with the thread parameters obtained without considering the axis angle, the final error was reduced by more than $50 \%$. On the parameter of pitch, the improvement effect of the thread parameter measurement by this algorithm was not obvious.

\section{CONCLUSION}

In this paper, an algorithm for profile correction of cylindrical external threads based on monocular vision is proposed. The influence of the inclination of thread axis on profile correction of cylindrical external threads is considered. When the angle between the cylindrical external thread axis and the $Z$ axis of the three-dimensional space coordinate system is at a certain angle, the image boundary equation of the thread cross-axis section and the difference equation of the thread profile can be obtained. The image boundary of cylindrical external thread obtained by the CCD camera is corrected by the difference equation feedback. A set of thread boundary detection system with uniform rotation of worktable is built to verify this algorithm, which improves the accuracy of thread quality detection. Limited by the experimental conditions, this paper uses the image boundary of approximate extremum position instead of the thread image boundary for calculation. Although the accuracy of thread parameter detection has been greatly improved, there are still some errors in the detection process. The proposed algorithm can be applied to further thread quality detection in industrial assembly lines.

\section{REFERENCES}

[1] Shen, M.H., Shen, S.F., Wang, X.C. (2011). Noncontact detection distortion correction of cylindrical thread type. Journal of Agricultural Machinery, 42 (10).

[2] Zhang, Z.F. (2013). Research on Non-contact Detection Technology of Conical Thread Based on Image Processing. Published Master's Thesis. Zhengjiang University, Hangzhou, Zhejiang, China. 
[3] Jiang, Z.P. (2018). Research on Non-contact Detection of Ball Screw Parameters Based on Image Processing. Published Master's Thesis. Zhengjiang University, Hangzhou, Zhejiang, China.

[4] Chen, S., Zhao, D., Lu, Y. (2013). A method for detecting alignment deviation on a thread-measuring instrument. Measurement Science and Technology, 24 (8), 085002.

[5] Min, J. (2015) Comprehensive measurement system for screw thread parameter based on machine vision. In 2015 International Conference on Computer and Computational Sciences (ICCCS). IEEE, 266-270.

[6] Claudinon, S., Lamesle, P., Orteu, J.J. (2002). Continuous in situ measurement of quenching distortions using computer vision. Journal of Materials Processing Technology, 122 (1), 69-81.

[7] Kosarevsky, S., Latypov, V. (2013). Detection of screw threads in computed tomography 3D density fields. Measurement Science Review, 13 (6), 292-297.

[8] Min, J. (2018). Measurement method of screw thread geometric error based on machine vision. Measurement and Control, 51 (7-8), 304-310.

[9] Manlong, C. (2018) Compensation of thread profile distortion in image measuring screw thread. Measurement, 129, 582-588.
[10] Erbao, P., Guotong, Z. (2012) Image processing technology research of on-line thread processing. Energy Procedia, 17, 1408-1415.

[11] Gadelmawla, E.S. (2017). Computer vision algorithms for measurement and inspection of external screw threads. Measurement, 100, 36-49.

[12] Hunsicker, R.J., Patten, J., Ledford, A. (2015). Automatic vision inspection and measurement system for external screw threads. Journal of Manufacturing Systems, 13 (5), 370-384.

[13] Zhang, H., Jin, G. (2019). Detection of machine visual external thread parameters based on SVM feature point classification. Sensors and Microsystems, 38 (04), 121124.

[14] Tong, Q.B., Jiao, C.Q., Huang, H. (2014). An automatic measuring method and system using laser triangulation scanning for the parameters of a screw thread. Measurement Science and Technology, 25 (3), 035202.

[15] Steger, C., Ulrich, M., Wiedemann, C. (2008). Machine Vision Algorithms and Applications (First Edition). Beijing, China: Tsinghua University Press.

Received June 27, 2019 Accepted January 25, 2020 